\title{
Measures of functional ability (disability) in arthritis in relation to impairment of range of joint movement
}

\author{
ELIZABETH M. BADLEY, SUSAN WAGSTAFF, AND PHILIP H. N. WOOD
}

From the ARC Epidemiology Research Unit, Manchester University Medical School

SUMMARY In the World Health Organisation's supplementary classification of the consequences of disease a distinction is made between impairments, such as are related to the site and nature of joint involvement, and any disabilities in everyday activities to which these may give rise. This paper considers the application of these ideas to people with arthritis by examining the extent of graded relationship between individual impairments, reflected by limitations in the range of joint movement, and the number or type of disabilities. Ninety-five people with three different types of arthritis were studied. A 41-item disability questionnaire was completed. Most of the variation was described by only 24 of the latter items. These fell into five broad functional groups-predominantly concerned with mobility, bending down, manual dexterity, bending the arm, and reaching above the head. The constituent activities could be scaled in order of difficulty of accomplishment. Aggregated scores for each of the functional groups were correlated with observed ranges of motion in relevant joints, and the ordering of difficulty was related to decreasing ranges of movement. These findings shed light on the genesis of disability and have implications for the development of more sensitive, specific, and simple methods of assessment in rheumatology. Appreciation of how disability relates to the localisation of disease manifestations provides a means for evaluating current methods of functional assessment and exposes potential biases in such appraisals.

Evaluation of the longer term outcome in rheumatoid arthritis, especially in relation to the effects of second-line drugs, is attracting considerable interest. Acute-phase phenomena such as the erythrocyte sedimentation rate (ESR), C-reactive protein (CRP), and articular index are of limited relevance. At least three meetings have been held in the last three years to consider functional measures in arthritis. ${ }^{1-3}$ Many scales have been based on activities of daily living (ADL) assessments, and the field has been reviewed by Liang and Jette. ${ }^{4}$ 'Quality of life' also features in the Arthritis Impact Measurement Scale (AIMS ${ }^{5-7}$ ) and the Health Assessment Questionnaire $\left(\mathrm{HAQ}^{89}\right)$, which have been used fairly widely in the United States; the latter has recently been adapted for use in the UK. ${ }^{10}$ However, very little effort has been expended on looking at the meaning of the scales or at their relation to

Accepted for publication 9 February 1984 .

Correspondence to Dr E. M. Badley. ARC Epidemiology Research Unit. University of Manchester Medical School. Stopford Building, Oxford Road, Manchester M13 9PT. the underlying disease process-that is, biological significance.

One approach is to exploit the model put forward by the World Health Organisation. ${ }^{11}$ This suggests that there is a progression: disease leads to impairment and thence to disability, and either can then give rise to handicap or disadvantage. Impairment in arthritis is reflected by pain and restriction in the range of movement of joints, whereas disability is expressed by difficulty or inability in performance of daily living activities. Exploration of this model ${ }^{12} 13$ suggested that disability in arthritis is to be related to the site and nature of involvement of the joints. Disabilities in different activities involving similar types of action or use of the same parts of the body tend to be found together, and the degree of disablement relates to the extent of impairment.

To test these ideas more rigorously the relationship between the extent of individual impairments and the number or type of disabilities has been studied. This paper describes our work in patients with rheumatoid arthritis and osteoarthritis. Although our primary concern has not been de- 
velopment of better measures of functional performance, the findings do shed light on the way assessments might be derived and used, particularly for monitoring progression of the underlying disease process.

\section{Materials and methods}

The study population consisted of 95 people currently attending hospital either as an inpatient or outpatient, all of whom were examined by one of us (S.W.). Forty patients had rheumatoid arthritis (RA), 39 had localised osteoarthrosis (LOA) in either the hip or knee of one or both limbs, and 16 had generalised osteoarthrosis (GOA) with some involvement of the upper as well as the lower limb. None suffered from any significant condition apart from arthritis. Their ages ranged from 28 to 84 years, with a mean of 61 (56 for those with RA, 64 for LOA, 65 for GOA); 63 were female.

The joints examined are indicated in Table 2 . Range of movement was measured with a goniometer for all recognised planes, the starting positions of the American Academy of Orthopedic Surgeons being used. ${ }^{14}$ Disability was ascertained by a 41-item disability questionnaire, which encompassed most of the activities considered in ADL assessments. Items were scored on the WHO disability severity scale, ${ }^{11}$ augmented to include a category for performance in an abnormal manner; the latter included modification of the customary way an activity had been carried out prior to onset of disease. This 8-point scale allows more sensitive grading of disabilities. Performance of each activity was scored as follows: 0 if no difficulties were encountered, 1 for difficulty, 2 for abnormal performance, 3 if aids were required, 4 for aids with a helping hand, 5 for personal assistance, 6 for personal help plus an aid, and 7 for activity impossible. Where questionnaire responses seemed discordant with the observed state of the joints, respondents were asked to demonstrate performance of the activity. In most of these instances the respondent had adapted the way in which an activity was performed to accommodate difficulties caused by the disease; this had been incorporated into personal activity patterns so as not to be regarded as unusual, though we scored it as such.

Factor analysis in earlier studies ${ }^{13}$ had shown that disabilities could be grouped according to functional

Table 1 Scaling properties of five functional disability groups and relation to overall disability score

\begin{tabular}{|c|c|c|c|c|}
\hline \multirow{2}{*}{$\begin{array}{l}\text { Functional } \\
\text { group }\end{array}$} & \multirow{2}{*}{$\begin{array}{l}\text { Constituent } \\
\text { activities* }\end{array}$} & \multirow{2}{*}{$\begin{array}{l}\text { Coefficient of } \\
\text { correlation with } \\
\text { aggregated score }\end{array}$} & \multicolumn{2}{|l|}{ Coefficients of } \\
\hline & & & Reproducibility & Scalability \\
\hline Mobility & $\begin{array}{l}\text { Walk to toilet, } \\
\text { get on and off toilet, } \\
\text { get in and out of bed, } \\
\text { turn side to side in bed, } \\
\text { go up and down stairs. } \\
\text { get in and out of bath. }\end{array}$ & 0.67 & 0.99 & $(0 \cdot 94$ \\
\hline Bending down & $\begin{array}{l}\text { Put on shocs and tic laces, } \\
\text { pull on socks/stockings/tights, } \\
\text { wash below waist-fect and towel dry, } \\
\text { cut toe/finger nails }\end{array}$ & $0 \cdot 72$ & 0.98 & $0 \cdot 89$ \\
\hline Dexterity & $\begin{array}{l}\text { Unscrew lid from } 1 \text { inch }(2.5 \mathrm{~cm}) \text { diam. jar, } \\
\text { prepare vegetables, } \\
\text { carve meat/slice bread. } \\
\text { cut toe/finger nails }\end{array}$ & $0 \cdot 82$ & 0.96 & 0.75 \\
\hline Bending arm & $\begin{array}{l}\text { Drink from full cup/beaker, } \\
\text { shave/apply cosmetics, } \\
\text { wash face and neck and towel dry, } \\
\text { wash trunk and arms and towel dry }\end{array}$ & $0 \cdot 80$ & 0.98 & 0.73 \\
\hline Reaching up & $\begin{array}{l}\text { Clothes over head, } \\
\text { brush/comb hair (esp. back), } \\
\text { wash hair. } \\
\text { put in electric plug shoulder height, } \\
\text { peg out washing. } \\
\text { use shelves situated above shoulder height. } \\
\text { open high window/clean window }\end{array}$ & 0.87 & 0.95 & $(0.69$ \\
\hline
\end{tabular}

Listed in increasing order of difficulty within each group. 
similarities in regard to the part of the body and kind of movements required. From this, and on patterns of associations in a correlation matrix, we constructed analogous groups, five of which we anticipated might scale. These are shown in Table 1, the constituent activities in the groups being predominantly concerned with mobility, bending down, manual dexterity, bending the arm, and reaching above the head. Cutting toe/finger nails appears in both the bending-down and dexterity groups, reflecting the different aspects of this composite activity.

A Guttman scaling technique was also used. A Guttman scale is one in which activities are arranged in order of difficulty, such that inability to perform one activity means that it would be impossible to carry out all the more difficult activities in the scale, and that no difficulty would be encountered with easier ones. Two statistics are associated with these scales. The coefficient of reproducibility is a measure of the proportion of all disabilities correctly predicted from the number of disabilities contributing to the scale that are shown by each case; satisfactory values conventionally equal or exceed 0.90 . The coefficient of scalability estimates the proportion of predictions, excepting the first item in the scale, found to be correct on the basis of the hypothesised sequence alone; satisfactory values conventionally equal or exceed $0 \cdot 60 .{ }^{15}$ Scalability is the more rigorous of the two coefficients, and so, in the presentation of the results, the latter have been ordered in terms of this coefficient. Guttman scaling requires that variables be binary, which means that scores have to be couched in terms of yes or no. Disabilities were dichotomised between major physical assistance or impossibility on the one hand, and use of aids, difficulty, or absence of problems on the other-that is between points 4 and 5 on the modified WHO scale.

An aggregated disability score for each functional group was computed by adding together the severity gradings of each constituent activity. Productmoment correlation analysis was used to relate this aggregated score to the range of motion of individual joints. Activity performance within each functional group was related to thresholds of movement in terms of both the maximum range at which the activity was found to be impossible and the minimum range at which a respondent had no difficulty performing the activity; all respondents with less than the minimum necessary range experienced at least some difficulty. The thresholds were non-exclusive in the sense that individuals with more than the minimum range could nevertheless still have a particular disability, and equally an individual with less than the maximum range could nevertheless find an activity impossible. This arises because ability to carry out a given activity is of course affected by problems with other joints. Despite their arbitrariness the thresholds formulated could be regarded as representing the critical limiting ranges for performance.

\section{Results}

The five functional groups shown in Table 1 include only 24 out of the original 41 disabilities. The overall summed disability score for these 24 activities accounted for $96 \%$ of the variation in the summed score for the full schedule (Pearson's $r=0.98 ; r^{2}$ is a measure of the variation accounted for by the correlation). The amount of variation in the overall score accounted for by each functional group ranged between 45 and $76 \%$ (based on $r^{2}$ ). The combined score for three groups, mobility, dexterity, and reaching up, had a correlation of 0.97 with the total summed score, implying that these three groups alone accounted for $94 \%$ of the variation in the total score. These three functional groups contain activities involving use of all the major joints of the body.

Table 1 also shows that the Guttman scaling coefficients for all five functional groups exceeded conventional minimum levels, indicating that the constituent activities shown form a cumulative scale in order of difficulty. One interpretation of these scaled groupings of disabilities is that they reflect problems with particular joints or groups of joints. This is supported when the overall score for the disability groupings is related to range of joint movement (Table 2). All correlations were negative, and for clarity only correlations of -0.4 or less are shown; in fact the remaining coefficients were appreciably less negative. With the size of sample studied, all correlations less than -0.3 are significant at the $0 \cdot 1 \%$ level or below. In this context, however, it is not so much statistical significance that is relevant as the magnitude of the variation accounted for. Correlations of the order of $0 \cdot 3$ account for only about $10 \%$ of the variation, whereas tıose shown in normal type in Table 2 account for $25 \%$ or more.

The mobility group correlated with range of flexion at the knee joint, and the bending group with range of hip flexion, though both the coefficients were on the low side. It is surprising that the hip showed no meaningful correlation with mobility. However, given the nature of the sample, of which over $40 \%$ had RA, only a low proportion of respondents had hip involvement. The reasonably high correlations of scores for the dexterity, bending arm, and reaching-up groups with flexion of joints in the hand might have been expected, as all activities 
Table 2 Relationship between functional disability groups and ranges of motion of relevant joints

\begin{tabular}{|c|c|c|c|c|c|c|c|c|c|c|}
\hline \multirow{2}{*}{$\begin{array}{l}\text { Functional } \\
\text { group }\end{array}$} & \multicolumn{10}{|c|}{ Correlation coefficients between range of joint motion and scores for functional groups } \\
\hline & $\begin{array}{l}\text { Knee } \\
\text { flexion } \\
\text { (1) }\end{array}$ & $\begin{array}{l}\text { Hip } \\
\text { flexion } \\
\text { (I) }\end{array}$ & $\begin{array}{l}\text { Shoulder } \\
\text { abduction }\end{array}$ & $\begin{array}{l}\text { Elbow } \\
\text { supination }\end{array}$ & $\begin{array}{l}\text { Wrist } \\
\text { extension }\end{array}$ & $\begin{array}{l}\text { Thumb } \\
\text { circum- } \\
\text { duction } \\
\text { (2) }\end{array}$ & $\begin{array}{l}\text { Thumb } \\
\text { extension }\end{array}$ & $\begin{array}{l}M C P \\
\text { flexion } \\
(3)\end{array}$ & $\begin{array}{l}\text { PIP } \\
\text { flexion } \\
\text { (3) }\end{array}$ & $\begin{array}{l}\text { DIP } \\
\text { flexion } \\
\text { (3) }\end{array}$ \\
\hline Mobility & $0 \cdot 56$ & & & & & & & & & \\
\hline $\begin{array}{l}\text { Bending down } \\
\text { Dexterity }\end{array}$ & & 0.53 & 0.42 & & & 0.63 & 0.65 & 0.51 & & 0.61 \\
\hline Bending arm & & & $0 \cdot 70$ & 0.51 & 0.58 & 0.58 & 0.59 & $0 \cdot 60$ & 0.47 & 0.55 \\
\hline Reaching up & & & 0.78 & & $0 \cdot 55$ & $0 \cdot 58$ & $0 \cdot 56$ & 0.51 & $0 \cdot 46$ & $0 \cdot 50$ \\
\hline
\end{tabular}

"Pearson's r: all correlations were negative, but the minus sign is not shown. Reported coefficients relate to: (1) the most limited side, (2) movement in the dominant limb, (3) the most limited joint on the dominant hand, on the assumption that these would represent the limiting difficulties in performance of most activities.

$\mathrm{MCP}=$ metacarpophalangeal joints. $\mathrm{PIP}=$ proximal interphalangeal joints. $\mathrm{DIP}=$ distal interphalangeal joints.

in these groups involved use of the hands. Correlations with the finger joints were highest for the dexterity group. The bending-arm group was the only one to show a meaningful correlation with any plane of elbow movement. Shoulder abduction was related to bending the arm and reaching above the head, particularly to the latter.

Not only were aggregated group scores related to joint movement, but this ordering was also associated with decreasing range of motion in the relevant joints. This is illustrated most clearly by threshold ranges (Table 3); by and large these followed the ordering of activities in the functional groups. Most of the deviations from the ordering can be explained by difficulties experienced with other joints, in some cases due to surgical fixation. Many activities showed very little variation between maximum and minimum ranges. This seemed to be a function of the nature of the joint and limitations in other joints.

The results presented so far have been pooled. Table 4 shows the Guttman scaling coefficients when RA, LOA, and GOA were considered separately. Scalability is considerably reduced by nonscale types, cases that do not fit in with the ordering of the scale, but most of the coefficients exceed the conventional minimum level. A scalability coefficient of 1.0 was found for LOA and GOA in the mobility and bending-down groups as all the cases which scored on the constituent activities fell on to the scale; for the bending-arm group few patients with these conditions scored on the relevant activities. Low coefficients were found in GOA for the dexterity and reaching-up groups, indicating that there was no satisfactory scaling in the small number of cases studied. Reaching up has been shown in two ways in Table 4 . Although the previously noted ordering was optimum for the whole sample, a better scale for RA has also been included with 'wash hair' being assigned after 'peg out washing' instead of immediately following 'brush/comb hair'.

\section{Discussion}

Most of the functional measures suggested for assessment of patients with arthritis consist of aggregated scores on a series of disabilities, such as those based on performance of activities of daily living (ADL assessments). Some workers seem to have more rationale than others in the choice of activities included in the scales, but all are to a greater or lesser extent arbitrary. There has been what amounts to an obsession with problems of reliability, to the virtual neglect of validity or biological significance. Measurement characteristics, such as test-retest reproducibility and intra- and interobserver variation, feature prominently in discussion of such scales. Overall scores have been reported as showing some alterations with disease progression, but the significance of such changes is often difficult to determine. In the absence of any external criteria, questions of validity have been subsumed into concern with reliability and correlations with other measures of the disease.

The problem has been lack of a reference standard of functional performance which could be used as a yardstick by which to judge assessment measures. It is difficult to conceive what a standard might consist of, because there is uncertainty over the meaning of functional performance. Our studies of the way disability develops in relation to an underlying disease process are intended to shed some light on this in the hope of providing a less arbitrary basis for functional assessment. 
Measures of functional ability (disability) in arthritis

$\eta=00000000000 m$

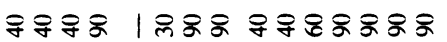

yo00 00no 00no00m

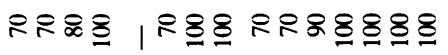

no00 00 ho oonooog

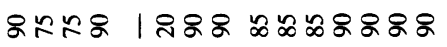

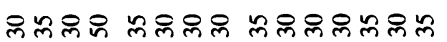

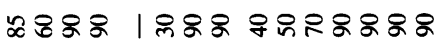

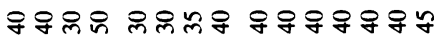

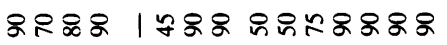

요이 00000000000

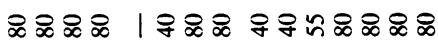

주으ำ

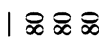

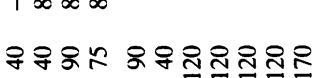

|

유ำสำ

ฐิฐิฐิ

マッッッきる

$1 \mid 1=00$

تृ

远

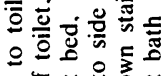

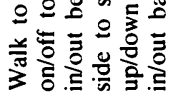
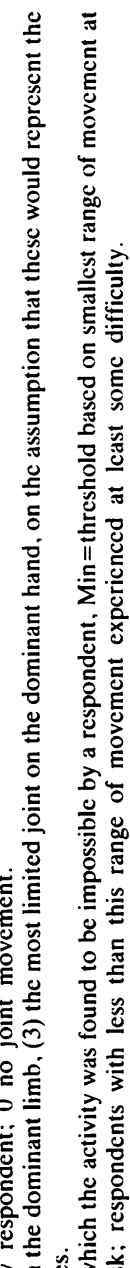

공.

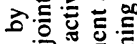

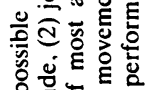
을

N

N

ర్

잉

○

훙

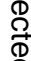

$\frac{0}{2}$ 
Table 4 Guttman scaling coefficients for functional disability groups according to type of arthritis

\begin{tabular}{|c|c|c|c|c|c|c|}
\hline \multirow{3}{*}{$\begin{array}{l}\text { Functional } \\
\text { group }\end{array}$} & \multicolumn{6}{|c|}{ Coefficients of } \\
\hline & \multicolumn{3}{|c|}{ Reproducibility } & \multicolumn{3}{|c|}{ Scalability } \\
\hline & $R A$ & $\angle O A$ & $G O A$ & $R A$ & $\angle O A$ & $G O A$ \\
\hline Mobility & 0.99 & $1 \cdot 00$ & $1 \cdot 00$ & 0.92 & $1 \cdot 00$ & 1.00 \\
\hline Bending down & 0.99 & 0.96 & 1.00 & 0.94 & 0.75 & $1 \cdot(0)$ \\
\hline Dexterity & 0.96 & 1.00 & 0.89 & 0.78 & 0.81 & 0.43 \\
\hline Bending arm & 0.96 & 1.00 & 1.00 & 0.68 & 1.00 & 1.00 \\
\hline \multirow{2}{*}{ Reaching up: 1} & 0.90 & 0.98 & 0.94 & 0.61 & 0.77 & $0 \cdot 33$ \\
\hline & 0.94 & 0.97 & 0.92 & 0.80 & 0.53 & $0 \cdot 11$ \\
\hline
\end{tabular}

Reaching up: $1=$ ordering of component disabilities as in Tables 1 and 3; $2=$ alternative ordering: clothes over head; brush/comb hair; plugs, shoulder height; peg out washing; wash hair; high shelves: open/clean high window.

Disability scaling has been reported before, but in more global contexts. Williams and subsequent workers ${ }^{16-19}$ found a range of basic activities, from being unable to eat without assistance to inability to use public transport, that could be scaled in order of difficulty. Various theories have been offered, including a 'rational choice theory' ${ }^{17}$ suggesting that the ordering reflected the choices people made about relinquishing performance of activities as they became more disabled. However, the setting for this work was concerned with overall scales of disablement and was without reference to underlying disease processes.

The work reported in this paper is of a different nature. It is more finely focused and considers a more closely integrated range of disabilities and underlying conditions. No attempt has been made to scale activities overall; any scaling is related to loss of range of movement. In our analyses we chose to start with disabilities, seeing to what extent these could be understood in terms of impairments-that is, the mapping is from activity restrictions to functional limitations. While the latter need not give rise to disability, it is a reasonable inference that the major part of such problems at least should be attributable to changes wrought by the underlying disease processes, as all disabilities must have some antecedent cause.

It is unreasonable to expect that one could completely explain the occurrence of disabilities by limitations in the range of joint movement. Pain and stiffness may exert independent effects, and fatigue and psychosocial factors such as 'drive' might influence the performance of activities. Indeed the correlations found between range of motion and scores for each functional disability group are not particular high, accounting at most for only just over half the variation (Table 2). It should be borne in mind, however, that our disability assessment relied on self-reported performance, and in our analyses we were unable to take other factors into account. Under these circumstances it is perhaps surprising that so much of the variation was explicable in terms of impaired ranges of joint motion.

Superficially it may appear that most assessment measures include a similar range of activities. However, because activities and disabilities are associated with use of different parts of the body, the overall score from a functional assessment could be biased if derived preferentially from activities concerned with one set of joints rather than another. For example, functional assessment in a multicentre trial of penicillamine was based on many items involving manual dexterity, and relatively few concerned with mobility and transfer. ${ }^{13-20}$ Variation in the way the upper limb was affected could therefore exert an undue effect on the overall score on such a scale. In contrast, the HAQ scale contains a majority of items concerned with mobility and transfer, and fewer related to manual dexterity. ${ }^{89}$ Changes in the total score on this scale would therefore be likely to be biased by the state of large joints in the lower limb. A relatively low correlation between the HAQ score and articular index might therefore be expected, in view of the relatively high contribution of the small joints of hands and feet to the latter.

The relationship between disability scales and underlying impairments may have other consequences for assessment. By pursuing this reasoning it is possible that one might be able to extend understanding back to more immediate manifestations of disease, such as $x$-ray or other changes. Such an approach might provide tools for studying the outcome of therapy with so-called disease-modifying drugs. Greater knowledge of the relationship between impairments and disabilities should also help elucidate the meaning of changes in overall disability scores, so as to eliminate 'noise' deriving from changes in disablement due to non-specific reasons, such as general ill health.

Grouping disabilities together, in terms of the parts of the body or the type of actions likely to be involved in activities, yields scores related to loss of range of movement in relevant joints. That such functional groups are linked to major joints suggests that disability assessments for localised conditions, such as OA of the hip or a frozen shoulder, could be streamlined by selecting those appropriate to the condition under investigation rather than using more global functional measures. For polyarticular conditions where there is preferential disease of 
different joints, sentinel joints could be selected for assessment.

The ordering of disabilities within scales seems to be parallel to the loss of range of movement. If this closely reflects the order in which ability to perform activities is gained or lost with improving or deteriorating range of movement, these results would have interesting implications for monitoring disease progression in arthritis. Rather than use an overall score, one could focus on the order in which disabilities are lost or gained. Obviously longitudinal studies would be needed to explore this possibility, and allowance would have to be made for the polyarticular nature of the arthritis.

We should also emphasise that the constituent activities in our functional groups comprise only a small proportion of the range included in a conventional ADL assessment. Our results suggest that for rheumatological evaluation a shortened assessment of disability might be possible, using activities occurring in only the mobility, manual dexterity, and reaching-up groups. By utilising the Guttman scaling properties within these groups an assessment of functional level could, in principle, be made from a starting point of only three questions. However, more work is required to optimise the scales for the different types of arthritic complaint, including more exploration of the choice of disability items to include.

Our thanks are due to Mr Andrew Lake for his considerable contribution to the carrying out of the analyses reported in this paper, and Miss Hilary Finegan for assistance with the preparation of the tables.

\section{References}

1 Paulus H E, Ehrlich G E, Lindenlaub E, eds. Controversies in the clinical evaluation of analgesic-anti-inflammatoryantirheumatic drugs. Symposia Medica Hoechst 16. Stuttgart: F K Schattauer, 1981.

2 Buchanan W W, Tugwell P, Bombardier C, eds. Conference on outcome measures in rheumatological clinical trials, Hamilton, December 1981. J Rheumatol 1982; 9: 749-806.
3 Kirwan J R, Chaput de Saintonge D M, Joyce C R B, Currey $\mathrm{H} \mathrm{L} \mathrm{F}$, eds. Advances in assessing rheumatoid arthritis. $\mathrm{Br} J$ Rheumatol 1983; 22 (suppl): 1-97.

4 Liang M, Jette A M. Measuring functional ability in chronic arthritis. Arthritis Rheum 1981; 24: 80-6.

5 Meenan R F, Gertman P M, Mason J H. Measuring health status in arthritis: the arthritis impact measurement scales. Arthritis Rheum 1980; 23: 146-52.

6 Meenan R F. Assessing therapeutic effectiveness in the rheumatic diseases: the case for functional and health status measures. In (1), pp 353-364.

7 Meenan R F, Gertman P M, Mason J H, Dunaif R. The arthritis impact measurement scales: further investigations of a health status measure. Arthritis Rheum 1982; 25: 1048-53.

8 Fries J F, Spitz P, Kraines R G, Holman H R. Measurement of patient outcome in arthritis. Arthritis Rheum 1980; 23: 137-45.

9 Fries J F, Spitz P, Young D Y. The dimensions of health outcomes: the health assessment questionnaire, disability and pain scores. $J$ Rheumatol 1982; 9: 789-93.

10 Kirwan J R, Reeback J S. Using a modified Stanford health assessment questionnaire to assess disability in UK patients with rheumatoid arthritis. Ann Rheum Dis 1983; 42: 219-20.

11 World Health Organisation (WHO). International classification of impairments, disabilities, and handicaps. Geneva: WHO, 1980.

12 Badley E M. Descriptors of disease consequences-their development and application. Dissertion, University of Manchester, 1982.

13 Badley E M, Lee J, Wood P H N. Patterns of disability related to joint involvement in rheumatoid arthritis. Rheumatol Rehabil 1979; 18: 105-9.

14 Heck C V, Hendryson I E, Rowe C R. Joint motion: methods of measuring and recording. American Academy of Orthopaedic Surgeons, 1965.

15 Torgerson W S. Theory and methods of scaling. New York: J Wiley, 1965.

16 Williams R G A, Johnston M, Willis L A, Bennett A E. Disability: a model and measurement technique. Brit J Prev Soc Med 1976; 30: 71-8.

17 Williams R G A. Theories and measurement in disability. $J$ Epidemiol Commun Hlth 1979; 33: 32-47.

18 Bebbington A C. Scaling indices of disablement. Brit J Prev Soc Med 1977; 31: 122-6.

19 Williams R G A. The prediction of varying sequences of handicap: a new analysis of the British Government's survey of the handicapped. In: Henrard J C, ed. Santé Publique et Vieillissement. Colloques Inserm 1981; 101: 181-94. Paris: Editions Inserm.

20 Multicentre Trial Group. Controlled trial of (D)-penicillamine in severe rheumatoid arthritis. Lancet 1973; i: 275-80. 